\title{
AVALIAÇÃO SENSORIAL DE “CACHAÇA” ENVELHECIDA EM TONEL DE CARVALHO (QUERCUS SP.) IRRADIADO
}

\author{
Silva Júnior $\mathrm{LS}^{\star}$, Faria $\mathrm{JB}^{\star *}$, Bolini $\mathrm{HMA}^{\star \star *}$
}

\begin{abstract}
Resumo
O destilado da cana-de-açúcar ou "cachaça" é produzido por destilação do caldo de cana-de-açúcar fermentado. O processo de envelhecimento dessa bebida pode melhorar sua qualidade sensorial, mas não representa uma prática regular entre os produtores brasileiros em decorrência das razões culturais e financeiras. $\mathrm{O}$ objetivo com este trabalho foi verificar se a irradiação dos barris poderia ser uma alternativa para reduzir o tempo de envelhecimento desse destilado. Quatro amostras do mesmo destilado de cana-de-açúcar, bidestiladas ( $68 \%$ de álcool v/v), foram submetidas aos seguintes processos: cinco litros foram colocados em uma garrafa de vidro (controle); cinco litros foram envelhecidos em

\footnotetext{
* Doutor em Ciência de Alimentos pela Universidade Estadual de Campinas; Mestre em Alimentos e Nutrição pela Universidade Estadual Paulista Júlio de Mesquita Filho; Professor do Curso de Engenharia de Alimentos na Universidade Estadual de Feira de Santana; luiz.severo@gmail.com

** Doutor e Mestre em Ciências dos Alimentos pela Universidade de São Paulo; Professor do Departamento de Alimentos e Nutrição na Universidade Estadual Paulista; fariajb@fcfar.unesp.br

*** Doutora em Tecnologia de Alimentos pela Universidade Estadual de Campinas; Mestre em Alimentos e Nutrição pela Universidade Estadual Paulista Júlio de Mesquita Filho; Professora do Curso de Engenharia de Alimentos na Universidade Estadual de Campinas; hellini@fea.unicamp.br
} 
um tonel de carvalho; cinco litros de uma amostra previamente irradiada foram colocados em outra garrafa de vidro; e cinco litros foram envelhecidos em outro tonel de carvalho previamente irradiado. Durante o período de envelhecimento, foram coletadas amostras correspondentes a: 1, 15, 30, 60, 90, 120, 150 e 180 dias e submetidas à análise sensorial. A irradiação foi realizada por uma fonte de cobalto, e todas as amostras foram diluídas para $40 \%$ de álcool v/v. Os testes sensoriais foram realizados por 25 provadores para verificar a diferença global e a aceitação entre as amostras. Os resultados sensoriais revelaram que a amostra envelhecida no tonel de carvalho irradiado apresentou características de destilados envelhecidos em um tempo reduzido, indicando o potencial uso da irradiação na aceleração do processo de envelhecimento. Palavras-chave: Cana-de-açúcar. Envelhecimento. Irradiação. Análise sensorial.

\section{Sensorial evaluation of "cachaça" spirit during aging in irradiated oak (Quercus sp.) cask}

\section{Abstract}

The sugar cane spirit or "cachaça" is produced by distillation offermented sugar cane juice. The aging process of this spirit can improve its sensorial quality, but it doesn't represent a regular practice among Brazilians producers due cultural and financial reasons. The aim of this work was to verify if the irradiation of the casks could be an alternative to reduce the aging time of this spirit. Four samples from the same sugar cane spirit, distilled twice (68\% alcohol $v / v$ ), were submitted to the following process: five liters were put in a glass bottle (control); five liters were aged in a small oak cask; five liters of a previously irradiated sample were put in another glass bottle; and five liters were aged in another oak cask previously irradiated. During the aging period samples corresponding to: 1, 15, 30, 60, 90, 120, 150 e 180 days were collected and submitted to sensory analysis. The irradiation was performed by a cobalt source and all samples were diluted to $40 \%$ alcohol $v / v$. Sensorial tests were carried out by 25 judges to verify the global difference and acceptation among the samples. The sensorial results revealed that the sample aged in the irradiated oak cask showed characteristics of aged spirits in a reduced time pointing out the potential use of irradiation in accelerating the aging process. Keywords: Sugar cane. Aging. Irradiation. Sensory analysis.

\section{INTRODUÇÃO}

A aguardente de cana é uma bebida muito apreciada pelo seu aroma e sabor característicos; possui graduação alcoólica que varia entre 38 e $54 \%$ em volume, a $20^{\circ} \mathrm{C}$, e pode ser obtida do destilado alcoólico simples da cana-de-açúcar ou pela destilação do mosto fermentado de cana. ${ }^{1}$

Os principais estágios para a obtenção da aguardente de cana são: fermentação, destilação e, em alguns casos, o envelhecimento. A fermentação deve ser conduzida de forma a se obter o máximo de rendimento em álcool etílico e uma proporção harmônica de componentes secundários. Da mesma forma, a destilação deve ser efetuada de forma que o destilado possua o aroma e o sabor dos elementos naturais voláteis contidos no mosto fermentado ou formados durante a destilação. Em es- 
tudo que avaliou a influência do tratamento térmico do caldo de cana-de-açúcar suplementado com ureia e submetido à destilação dupla contendo os compostos voláteis (aldeído acético, acetato de etilo, n-propil, isobutilo e álcoois isoamílicos e ácido acético) e os contaminantes (metanol, 1-propilo e álcoois 2-butil, cobre e carbamato de etilo) na cachaça, verificou-se que os produtos destilados originados de alíquotas contaminadas apresentaram maior concentração de ácido acético e carbamato de etila e que a destilação dupla reduziu a concentração desses contaminantes. ${ }^{2}$

No estágio de envelhecimento da bebida observa-se que a qualidade sensorial da aguardente é nitidamente afetada em razão direta da permanência do produto em barris de madeira. As características de cor, aroma e sabor da bebida envelhecida evoluem de forma que estão relacionadas com o tipo de madeira e tratamentos prévios, à geometria e história do barril e ao tempo de estocagem. O envelhecimento é efetuado mantendo-se a aguardente em recipientes de madeira adequados e em condições próprias de armazenamento, principalmente em relação à temperatura e umidade. ${ }^{3}$

A etapa de envelhecimento da bebida não é obrigatória para a cachaça, porém, quando é realizada, os barris geralmente não passam pela chamuscagem final da madeira. No entanto, o nível de degradação térmica da madeira influencia a formação de novos compostos químicos, que são liberados durante o processo de envelhecimento e melhoram a qualidade do destilado. Estudos realizados comprovam que a geração de compostos marcadores de envelhecimento são influenciados apenas pela intensidade da chamuscagem da parte interna do barril e não pela origem da madeira de carvalho. ${ }^{4}$

Porém, informações sobre as modificações que ocorrem na aguardente de cana durante o envelhecimento ainda são limitadas. Entretanto, estudos conduzidos com uísque, conhaque, brandy e rum podem orientar de forma bastante precisa quanto às possíveis modificações químicas, físicas e sensoriais da aguardente de cana durante o envelhecimento. Esse paralelismo pode ser traçado com relativa segurança, em razão do comportamento similar ao uísque mostrado pelo conhaque, brandy e rum. ${ }^{5}$ Um estudo de análise sensorial com uísques escoceses, classificados em quatro categorias: luxo, padrão, comercial e padrão ocidental, a partir da análise de variância (ANOVA), atributos altamente significativos para a discriminação entre os uísques que foram misturados, apresentaram-se pungentes, fumegantes, lisos, amadeirados, com aroma de baunilha, sulfúricos e rançosos. ${ }^{6}$

Um dos métodos que pode acelerar o processo de envelhecimento é o tratamento por radiação ionizante. O processo envolve a exposição do material a uma quantidade controlada de radiação iônica, por um tempo determinado, para que sejam atingidos os objetivos esperados. A energia radiante ocorre de diversas formas e apresenta características próprias, relacionadas com sua frequência, comprimento de onda e potencial alcance. A irradiação de um material que contém água causa ionização de uma parte das moléculas, formação de hidrogênio altamente reativo e radicais hidroxilas. As radiações ionizantes têm utilização potencial no envelhecimento de vinhos e aceleram o processo de envelhecimento de uísques, modificando o sabor e a aparência do destilado, em razão das mudanças oxidativas que ocorrem de forma acelerada. $\mathrm{O}$ tratamento de irradiação pode, também, provocar alterações na madeira, como, por exemplo, a hidrólise da celulose. Em pesquisa sobre o efeito da irradiação (0-10 kGy) no perfil de aguardente de cana por cromatografia gasosa, em recipientes de vidro contendo raspas de carvalho, verificou-se que o efeito da irradiação é mais acentuado quando há presença da madeira irradiada no recipiente contendo a aguardente. 
O tratamento de alimentos por radiações ionizantes específicas para melhorar a segurança do produto e armazenamento é uma das tecnologias mais estudadas atualmente. No entanto, grande parte da pesquisa foi realizada em laboratórios e ainda é relativamente subutilizada comercialmente. O seu potencial de aplicação é muito diversificado, desde a inibição de brotos em tubérculos até a obtenção de produtos alimentícios comercialmente estéreis. A segurança do consumo e a salubridade dos alimentos irradiados têm sido amplamente estudadas em cooperações internacionais. Foi desenvolvido um Padrão Geral do Codex para alimentos irradiados e um Código de Prática Internacional recomendado para o processamento de radiação de alimentos. Aplicações específicas de irradiação de alimentos foram aprovadas pelas legislações nacionais em mais de 55 países em todo o mundo. ${ }^{8}$

As decisões nesses e em outros países foram influenciadas pela adoção, em 1983, de uma norma mundial envolvendo alimentos irradiados. Essa Norma foi adotada pelo Codex Alimentarius Comission, órgão associado à Food and Agriculture Organization of the United Nations (FAO) e à World Health Organization (WHO). Ela é baseada nas pesquisas da Joint Expert Commitee on Food Irradiation (JECFI), em associação com a FAO, WHO e International Atomic Energy Agency (IAEA), e permitiu concluir que a irradiação de qualquer alimento, até uma dose média de 10 kilogray, não apresenta riscos toxicológicos. Para facilitar a compreensão e corrigir os mitos sobre a irradiação de alimentos e bebidas, uma série de comunicados está sendo preparada pelo International Consulative Group on Food Irradiation (ICGFI). O ICGFI foi implantado pela FAO, IAEA e WHO para o aconselhamento de diferentes organizações visando resolver problemas relacionados ao comércio internacional, saúde pública, economia, regulamentação e informação à população. ${ }^{9}$

O uso comercial da tecnologia de irradiação de alimentos no Brasil cresce lentamente em decorrência das interpretações equivocadas por grande parte dos consumidores brasileiros, que possui uma ideia preconcebida, advinda da imagem negativa da energia nuclear. Pesquisas indicam que a difícil aceitação por parte do consumidor pode ser atribuída à confusão existente entre os termos irradiação e radioatividade, frequentemente relacionados aos riscos à saúde. Quando devidamente informados sobre o que é, para que serve e quais os benefícios da tecnologia de irradiação de alimentos, grande parte dos consumidores reage de forma positiva. ${ }^{10}$

O objetivo com este trabalho foi avaliar o efeito da irradiação prévia do ancorote de carvalho sobre o tempo de envelhecimento de aguardente de cana bidestilada por meio de testes sensoriais.

\section{MATERIAIS E MÉTODOS}

\subsection{MATERIAIS}

Amostras de um mesmo lote de cachaça bidestilada com graduação alcoólica de 68\% em volume, a $20^{\circ} \mathrm{C}$, foram submetidas a quatro tratamentos, conforme descreve-se na Figura 1.

As amostras foram armazenadas em ambiente com aproximadamente $70 \%$ de umidade, 28 ${ }^{\circ} \mathrm{C}$ de temperatura e ausência de luminosidade. 


\begin{tabular}{|c|l|}
\hline Amostras & \multicolumn{1}{|c|}{ Processos de Tratamento } \\
\hline A1 & Armazenada em recipiente de vidro (5 litros) (controle 1) \\
\hline A2 & Envelhecida em ancorote de carvalho (5 litros) (controle 2) \\
\hline A3 & Armazenada em recipiente de vidro (5 litros), utilizando uma amostra de cachaça irradiada \\
\hline A4 & Envelhecida em ancorote de carvalho (5 litros) previamente irradiado \\
\hline
\end{tabular}

Figura 1 - Processos de tratamento das amostras

O tratamento radioativo do ancorote e da amostra de cachaça foi realizado pela Empresa Brasileira de Radiação (Embrarad), que utilizou uma fonte de radiação $\gamma$ de cobalto 60. A amostra de cachaça ficou exposta a 0,3 kGy por 45 segundos, e o ancorote de carvalho a $185 \mathrm{kGy}$, por 27 horas, totalizando, nessa última exposição, nove ciclos.

Durante o período de estudo foram coletadas alíquotas correspondentes a 1, 15, 30, 60, 90, 120, 150 e 180 dias de armazenamento, que foram, então, diluídas com água destilada até 40\% de álcool em volume, a $20^{\circ} \mathrm{C}$, antes de serem analisadas sensorialmente.

\subsection{MÉTODOS}

\subsubsection{Análise sensorial - Teste de aceitação}

As amostras de aguardente foram submetidas à análise de aceitação em relação ao aroma, sabor, impressão global e cor por uma equipe de 25 provadores em cabines laboratoriais individuais, sendo utilizadas escalas hedônicas não estruturadas de nove centímetros. As amostras foram apresentadas de forma monádica em cálices vermelhos opacos, codificados com algarismos de três dígitos e cobertos com vidro de relógio que eram retirados no momento do teste. Nos testes de aceitação, em relação à cor, as amostras foram apresentadas em cálices codificados transparentes, sobre superfície branca, em blocos completos casualisados.

Foi realizado também um teste de diferença do controle (amostra A1) para verificar o grau de diferença das amostras A2, A3 e A4 em relação à amostra A1. Nesse caso, as amostras foram servidas em copos descartáveis e brancos, codificados com algarismos de três dígitos para as amostras A2, A3 e A4 e com a letra P para a amostra controle (ou padrão).

\subsubsection{Análise estatística}

Os resultados obtidos nos testes de aceitação foram avaliados por análise de variância univariada (ANOVA), testes de médias de Tukey $(\mathrm{p} £ 0,05)$ e por análise dos histogramas da frequência de distribuição das notas dadas pelos provadores em relação à escala utilizada para cada amostra.

Foi realizada também análise de regressão linear entre o tempo de envelhecimento e a aceitação para cada atributo avaliado nas amostras de aguardente. Os dados obtidos para os testes de 
diferença do controle foram analisados por ANOVA e testes de médias de Dunnett ( $\mathrm{p} £ 0,05)$. Essas análises foram realizadas utilizando-se o programa estatístico SAS. ${ }^{11}$

\section{RESULTADOS}

\subsection{ANÁLISE SENSORIAL}

\subsubsection{Teste de diferença do controle}

A partir do primeiro dia as amostras A2 (envelhecida em ancorote de carvalho) e A4 (envelhecida em ancorote de carvalho irradiado) já diferiram da amostra controle, e a análise de aceitação comprovou que essa diferença era positiva em relação à cor, pois a aceitação das amostras envelhecidas foi significativamente superior.

Em nenhum período a amostra A3 (cachaça irradiada) diferiu da amostra controle, comprovando que a irradiação diretamente na cachaça não promoveu alterações sensoriais.

Podemos afirmar que a amostra A1 (controle) não apresentou diferença significativa ( $\mathrm{p} £$ 0,05) no decorrer do tempo de armazenamento, considerando-se os atributos avaliados.

Os resultados da análise de regressão revelaram também que não houve correlação entre o tempo de armazenamento e as médias dadas pelos provadores quanto aos atributos avaliados para a amostra A1(controle), conforme Tabela 1. Esse fato pode ser explicado pelo próprio processo de armazenamento a que a amostra foi submetida, ou seja, em recipiente de vidro, mantendo o produto inalterado, sem percepção sensorial.

Tabela 1 - Médias para aceitação em função do tempo de armazenamento para a amostra A1 (controle) ${ }^{\dagger}$

\begin{tabular}{|c|c|c|c|c|}
\hline DIAS & AROMA & SABOR & IGL & COR \\
\hline 1 & $3,80^{\mathrm{a}}$ & $3,09^{a}$ & $3,48^{a}$ & $1,57^{\mathrm{a}}$ \\
\hline 15 & $3,33^{\mathrm{a}}$ & $3,13^{a}$ & $3,66^{\mathrm{a}}$ & $1,54^{\mathrm{a}}$ \\
\hline 30 & $3,56^{\mathrm{a}}$ & $3,09^{a}$ & $3,19^{a}$ & $1,31^{\mathrm{a}}$ \\
\hline 60 & $3,32^{\mathrm{a}}$ & $3,12^{a}$ & $3,24^{\mathrm{a}}$ & $1,10^{\mathrm{a}}$ \\
\hline 90 & $3,92^{\mathrm{a}}$ & $3,89^{a}$ & $3,94^{\mathrm{a}}$ & $1,04^{\mathrm{a}}$ \\
\hline 120 & $4,09^{\mathrm{a}}$ & $3,60^{\mathrm{a}}$ & $3,37^{a}$ & $1,05^{\mathrm{a}}$ \\
\hline 150 & $3,65^{\mathrm{a}}$ & $3,24^{\mathrm{a}}$ & $3,17^{\mathrm{a}}$ & $1,17^{\mathrm{a}}$ \\
\hline 180 & $3,56^{\mathrm{a}}$ & $3,39^{a}$ & $3,35^{\mathrm{a}}$ & $1,03^{\mathrm{a}}$ \\
\hline DMS & 1,19 & 1,30 & 1,38 & 0,59 \\
\hline
\end{tabular}

Observou-se, também, no caso da amostra A2, alta correlação linear positiva significativa ( $\mathrm{p}$ $£ 0,05)$ entre o tempo de envelhecimento e as médias dos provadores em relação a todos os atributos avaliados, em que R é o coeficiente de correlação linear de Pearson, conforme Figura 2.

\footnotetext{
${ }^{\dagger}$ Médias com letras iguais na mesma coluna não diferem entre si significativamente; DMS = diferença mínima significativa; IGL = impressão global.
} 


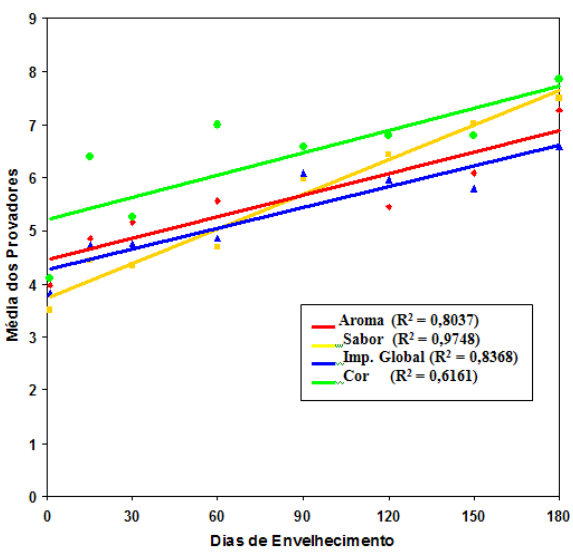

Figura 2 - Médias de aceitação para a amostra A2 (armazenada em ancorote de carvalho) em função do tempo de envelhecimento para os atributos avaliados

Com base nos resultados da ANOVA e nos testes de média de Tukey, observou-se que a amostra A3 (cachaça irradiada) não apresentou diferença significativa ( $\mathrm{p} £ 0,05$ ) ao longo do tempo de armazenamento em relação a todos os atributos avaliados (Tabela 2).

Tabela 2 - Médias para aceitação em função do tempo de armazenamento para a amostra A3 (cachaça irradiada) *

\begin{tabular}{rrrrr}
\hline DIAS & AROMA & SABOR & IGL & COR \\
\hline 1 & $3,79^{\mathrm{a}}$ & $3,25^{\mathrm{a}}$ & $4,04^{\mathrm{a}}$ & $1,34^{\mathrm{a}}$ \\
15 & $3,59^{\mathrm{a}}$ & $3,28^{\mathrm{a}}$ & $3,92^{\mathrm{a}}$ & $1,50^{\mathrm{a}}$ \\
30 & $3,64^{\mathrm{a}}$ & $3,09^{\mathrm{a}}$ & $3,35^{\mathrm{a}}$ & $1,24^{\mathrm{a}}$ \\
60 & $3,35^{\mathrm{a}}$ & $3,26^{\mathrm{a}}$ & $3,40^{\mathrm{a}}$ & $1,24^{\mathrm{a}}$ \\
90 & $3,97^{\mathrm{a}}$ & $3,71^{\mathrm{a}}$ & $4,37^{\mathrm{a}}$ & $1,15^{\mathrm{a}}$ \\
120 & $3,82^{\mathrm{a}}$ & $4,39^{\mathrm{a}}$ & $3,55^{\mathrm{a}}$ & $1,12^{\mathrm{a}}$ \\
150 & $3,30^{\mathrm{a}}$ & $3,35^{\mathrm{a}}$ & $3,35^{\mathrm{a}}$ & $1,28^{\mathrm{a}}$ \\
180 & $3,06^{\mathrm{a}}$ & $3,06^{\mathrm{a}}$ & $3,66^{\mathrm{a}}$ & $1,17^{\mathrm{a}}$ \\
DMS & 1,18 & 1,13 & 1,28 & 0,61 \\
\hline
\end{tabular}

Analisando-se os resultados do teste de aceitação para a amostra A4 (armazenada em ancorote de carvalho irradiado), observou-se uma tendência de aumento de aceitação, com o aumento do tempo de envelhecimento para essa amostra, que apresentou médias significativamente superiores ( $p$ $£ 0,05$ ) em relação a todos os atributos a partir de 30 dias (Tabela 3 ).

\footnotetext{
* Médias com letras iguais na mesma coluna não diferem entre si significativamente; DMS = diferença mínima significativa; IGL = impressão global.
} 
Tabela 3 - Médias para aceitação da amostra 4 (armazenada em ancorote de carvalho irradiado) em função do tempo de envelhecimento $\$$

\begin{tabular}{|c|c|c|c|c|}
\hline DIAS & AROMA & SABOR & IGL & COR \\
\hline 1 & $3,98^{\mathrm{d}}$ & $3,80^{\mathrm{e}}$ & $3,84^{\mathrm{b}}$ & $4,86^{c}$ \\
\hline 15 & $5,37^{c}$ & $4,36^{\mathrm{d}, \mathrm{e}}$ & $4,32^{\mathrm{b}}$ & $6,44^{b}$ \\
\hline 30 & $6,45^{\mathrm{c}, \mathrm{b}}$ & $5,49^{\mathrm{d}, \mathrm{c}}$ & $5,93^{\mathrm{a}}$ & $6,47^{b}$ \\
\hline 60 & $6,92^{\mathrm{a}}$ & $5,74^{\mathrm{b}, \mathrm{c}}$ & $5,98^{\mathrm{a}}$ & $7,43^{\mathrm{b}, \mathrm{a}}$ \\
\hline 90 & $7,31^{\mathrm{a}, \mathrm{b}}$ & $6,38^{a, b, c}$ & $6,74^{a}$ & $7,88^{\mathrm{a}}$ \\
\hline 120 & $7,84^{\mathrm{a}}$ & $6,20^{\mathrm{b}, \mathrm{a}}$ & $7,06^{\mathrm{a}}$ & $7,58^{\mathrm{a}}$ \\
\hline 150 & $7,16^{\mathrm{a}, \mathrm{b}}$ & $7,56^{\mathrm{a}}$ & $6,94^{\mathrm{a}}$ & $7,58^{\mathrm{b}, \mathrm{a}}$ \\
\hline 180 & $7,66^{\mathrm{a}}$ & $7,28^{a}$ & $6,78^{a}$ & $8,30^{\mathrm{a}}$ \\
\hline DMS & 1,18 & 1,28 & 1,32 & 1,29 \\
\hline
\end{tabular}

Observou-se, também, o aumento significativo da aceitação verificado nos primeiros três meses, seguido por uma estabilização das médias até o tempo final do experimento. É comprovado cientificamente que o conteúdo de aldeídos nas bebidas destiladas aumenta com os processos oxidativos. ${ }^{12}$ Esses processos de irradiação e envelhecimento são conhecidos como processos oxidativos, em que a exposição de amostras aquosas à radiação gama sob condições aeróbicas aumenta o potencial de óxido-redução do sistema, formando radicais livres que são compostos altamente reativos como o superóxido e radical hidroxila. ${ }^{13}$ Nesse sentido, verificou-se que os radicais livres formados pela interação da radiação com os compostos contidos nas bebidas analisadas induziram um fenômeno químico semelhante àquele que acontece por meio da lenta ação do tempo, ou seja, um aumento da concentração de aldeídos, uma das classes de substâncias responsáveis pelo flavor específico da bebida envelhecida.

A análise de regressão também mostrou alta correlação linear positiva significativa ( $\mathrm{p} £ 0,05)$ entre o tempo de envelhecimento e as médias dos provadores para todos os atributos avaliados, de acordo com a Figura 3.

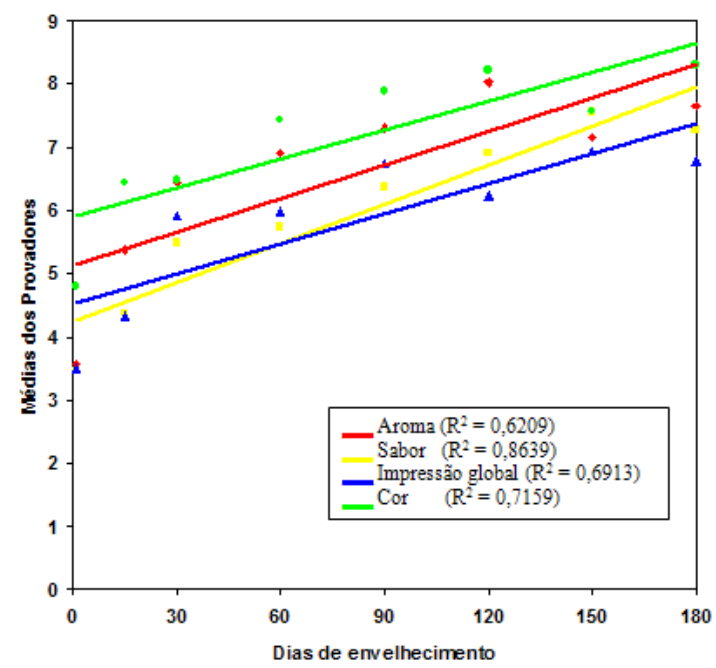

Figura 3 - Médias de aceitação para a amostra 4 (armazenada em ancorote irradiado) em função do tempo de envelhecimento para os atributos avaliados

\footnotetext{
$\S$ Médias com letras iguais na mesma coluna não diferem entre si significativamente; DMS = diferença mínima significativa; IGL = impressão global.
} 
No caso da amostra A2 (armazenada em ancorote de carvalho), as porcentagens de provadores em função das notas atribuídas para aroma, sabor, impressão global e cor foram representadas nas Figuras 4, 6, 8 e 10. Observou-se, nesse caso, uma tendência de um maior número de provadores atribuírem notas mais elevadas para todos os atributos avaliados no decorrer do período estudado.

Para a amostra 4 (envelhecida em ancorote irradiado), observou-se maior aceitação, considerando os atributos aroma e cor, que foram significativamente superiores, no período de 30 a 120 dias, em comparação com a amostra A2, submetida ao envelhecimento em ancorote isento de radiação, conforme Figura 4. De acordo com os resultados obtidos nos testes de aceitação, considerando as amostras A2 e A4 para os atributos avaliados observou-se que no período de 30 dias houve diferença estatisticamente significativa ( $\mathrm{f}$ 0,05) para aroma, sabor, impressão global e cor. Esses resultados favoráveis à amostra A4 (Figura 5) podem ser em razão do efeito da radiação no ancorote de carvalho, fenômeno também comprovado por estudo14 que investigou o efeito da radiação ultravioleta nas bordas e na parte interna do barril de carvalho no envelhecimento de brandy, sendo observada, em ambos os casos, a presença de compostos voláteis, como polifenóis, peróxidos orgânicos, furfural, alcoóis superiores, aldeídos e acetáis, importantes para o flavor de bebidas destiladas. Os pesquisadores sugeriram que o envelhecimento de, no mínimo, dois a cinco anos possa ser substituído por 50 horas de radiação ultravioleta sob condições próprias de umidade e em presença de ar.

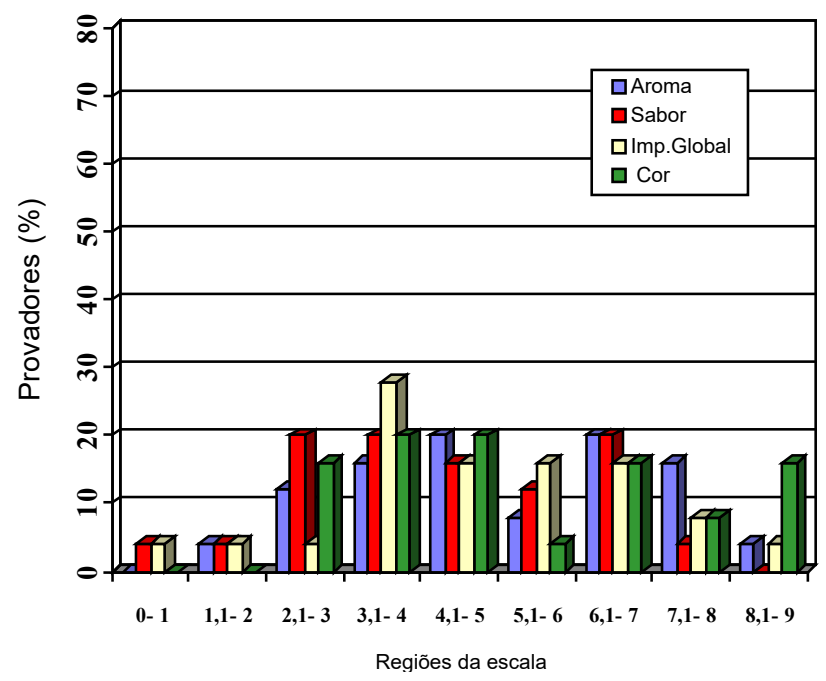

Figura 4 - Teste de aceitação da amostra A2 (armazenada em ancorote de carvalho) aos 30 dias de envelhecimento 


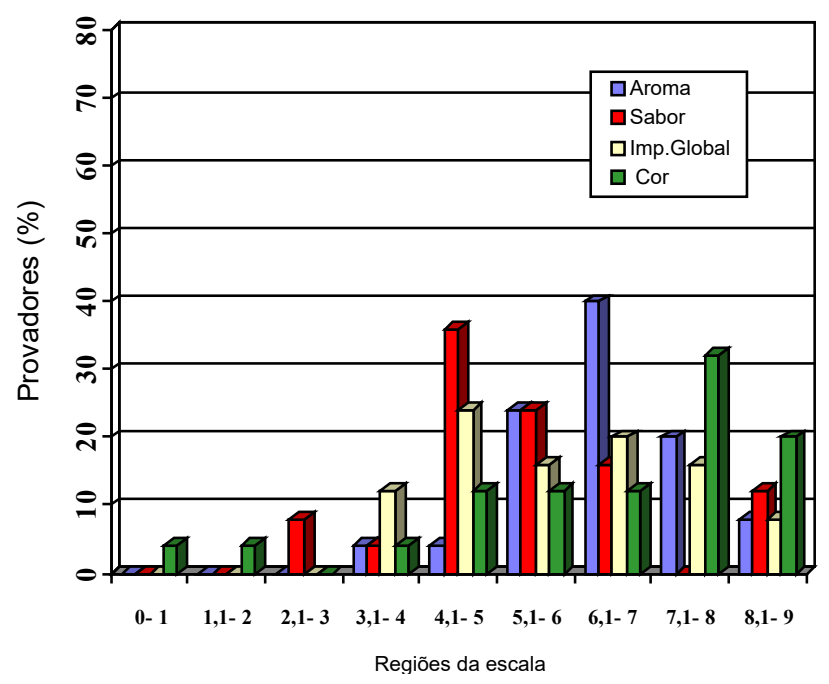

Figura 5 - Teste de aceitação da amostra A4 (armazenada em ancorote de carvalho irradiado) aos 30 dias de envelhecimento

Aos 60 dias de envelhecimento, observou-se uma diferença estatística significativa (p $£ 0,05)$ apenas para o aroma, considerando as amostras A2 e A4. Esses resultados estão representados nas Figuras 6 e 7.

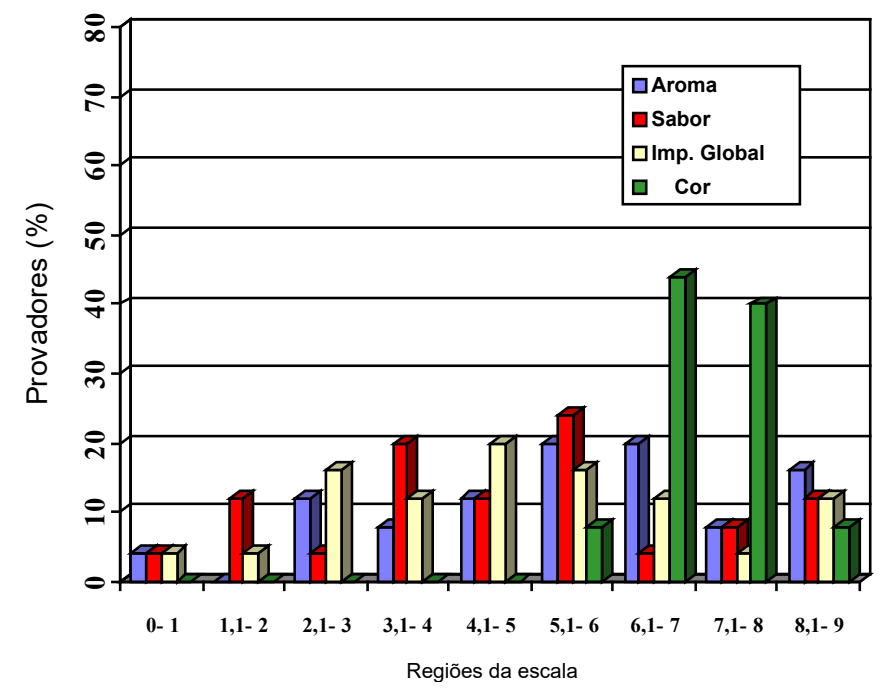

Figura 6 - Teste de aceitação da amostra A2 (armazenada em ancorote de carvalho) aos 60 dias de envelhecimento 


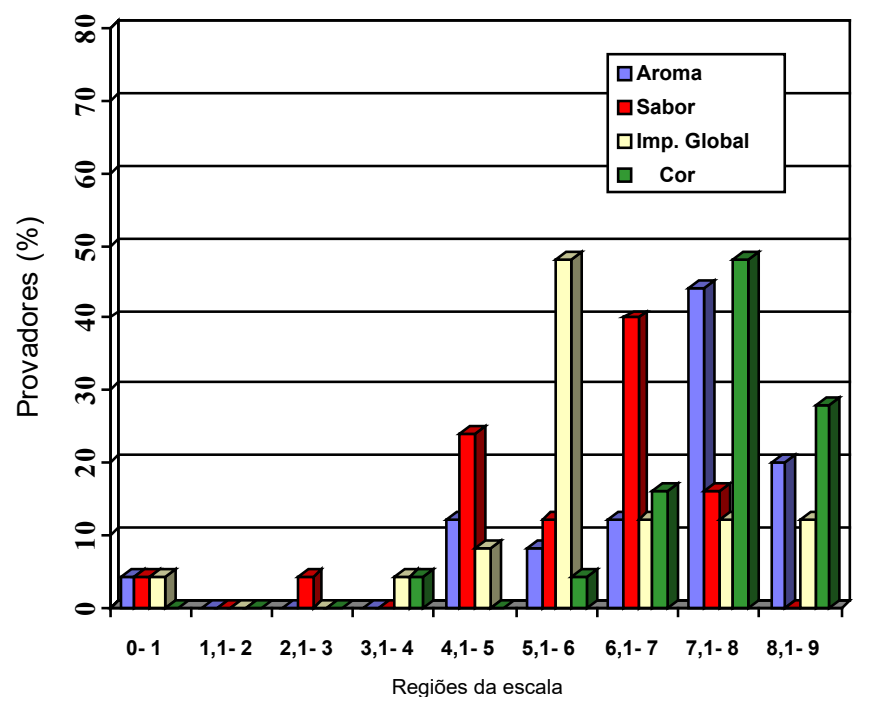

Figura 7 - Teste de aceitação da amostra A4 (armazenada em ancorote de carvalho irradiado) com 60 dias de envelhecimento

Os resultados do teste de aceitação para 90 dias de envelhecimento estão representados nas Figuras 8 e 9. Observa-se que apenas os atributos aroma e cor apresentaram diferença estatística significativa $(\mathrm{p} £ 0,05)$ para as amostras A2 e A4.

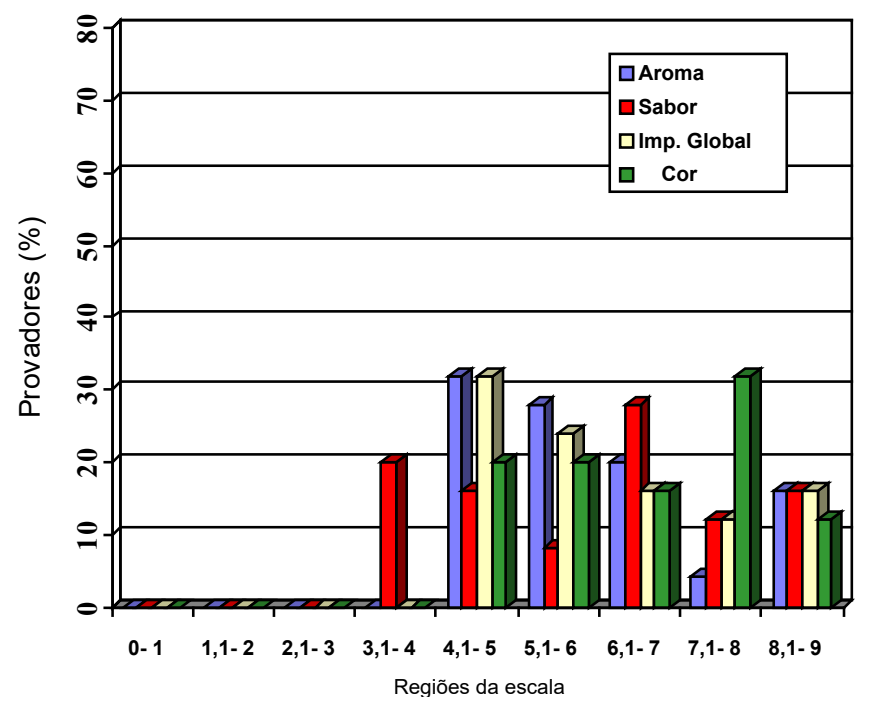

Figura 8 - Teste de aceitação da amostra A2 (armazenada em ancorote de carvalho) com 90 dias de envelhecimento 


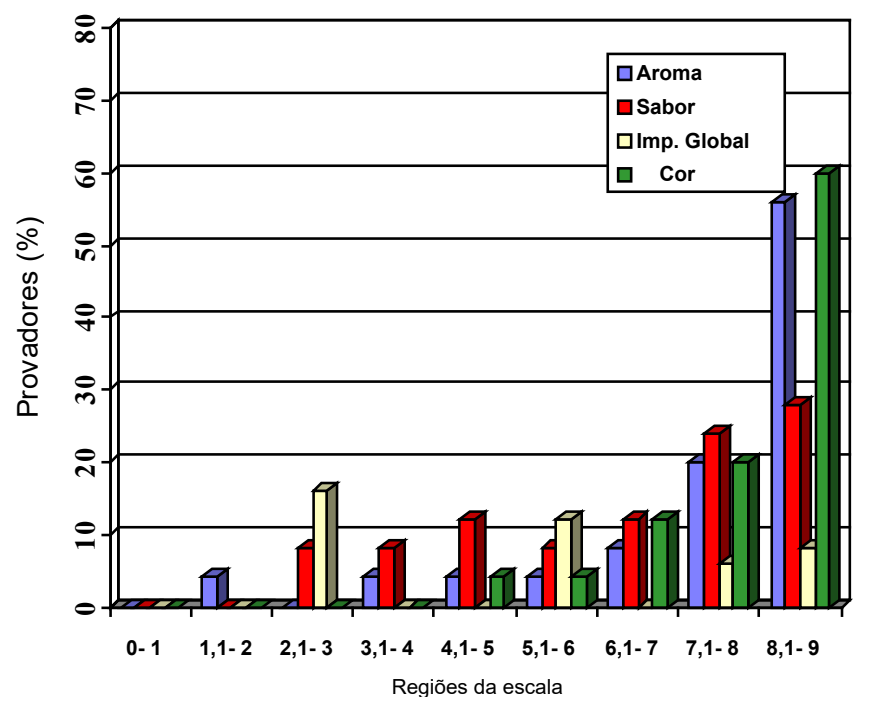

Figura 9 - Teste de aceitação da amostra A4 (armazenada em ancorote de carvalho irradiado) aos 90 dias de envelhecimento

Foi observada também diferença estatística significativa $(\mathrm{p} £ 0,05)$ para aroma e cor aos 120 dias de envelhecimento para as amostras A2 e A4. Esses resultados estão representados nas Figuras 10 e 11.

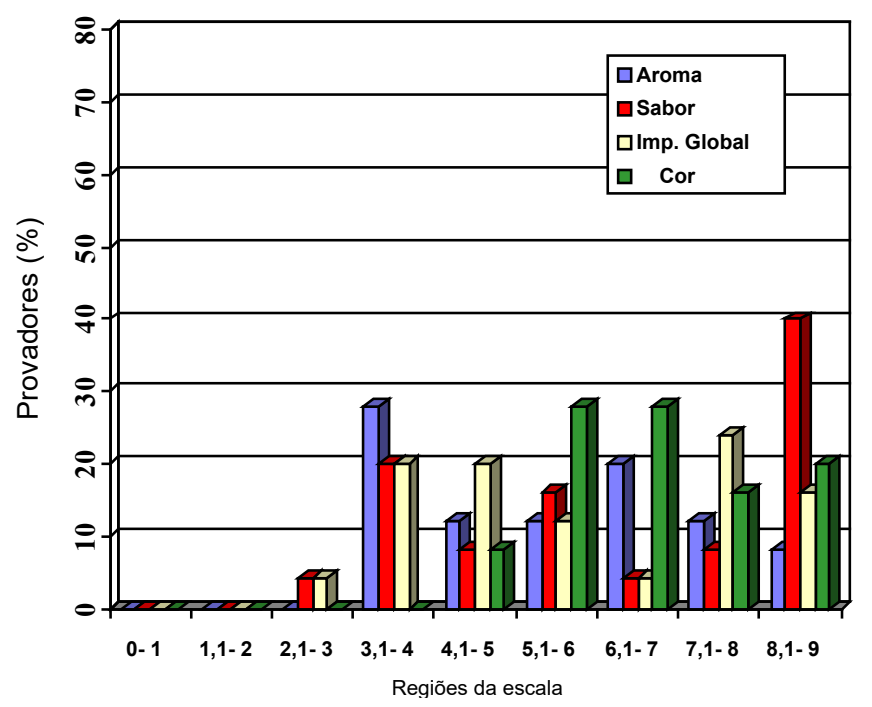

Figura 10 - Teste de aceitação da amostra A2 (armazenada em ancorote de carvalho) aos 120 dias de envelhecimento 


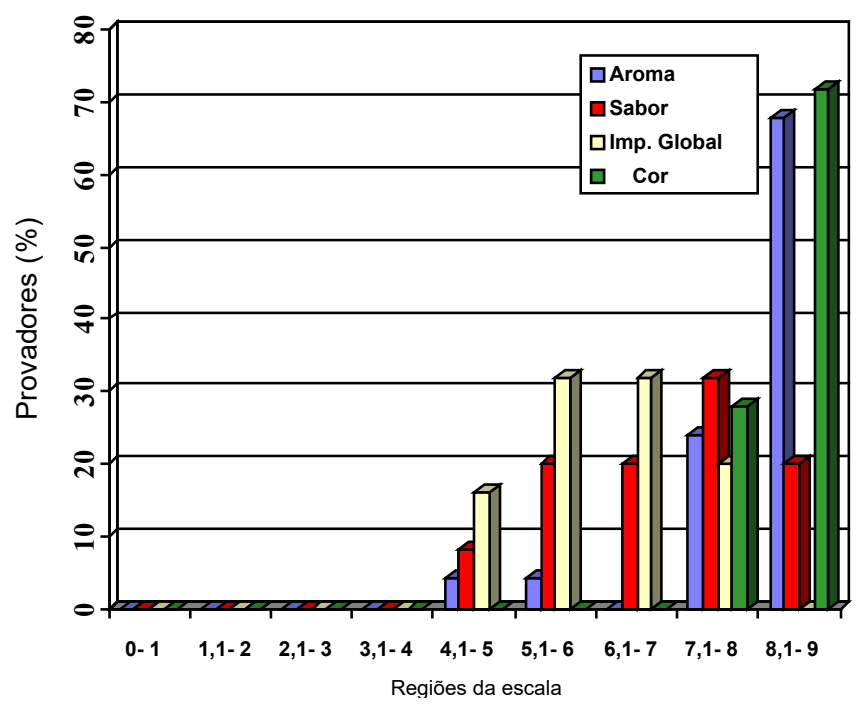

Figura 11 - Teste de aceitação da amostra A4 (armazenada em ancorote de carvalho irradiado) aos 120 dias de envelhecimento

A amostra A2, ao final de 150 dias de envelhecimento, obteve uma aceitação máxima em relação ao sabor, com 64\% da preferência com notas entre 7,1 e 8. Para aroma foram registradas notas na faixa de 8,1 a 9 por $28 \%$ dos provadores; para impressão global, o maior número de provadores (36\%) optou por notas entre 7,1 e 8,28\% dos provadores atribuíram notas entre 5,1 e 6, e 28\%, notas entre 6,1 e 7 para cor. Os resultados estão apresentados na Figura 12.

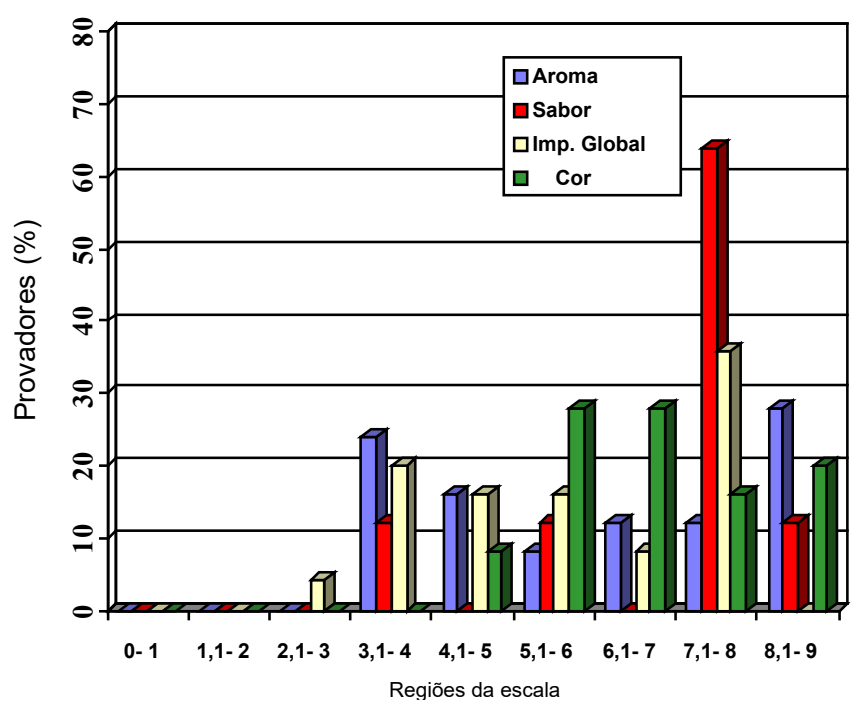

Figura 12 - Teste de aceitação da amostra A2 (armazenada em ancorote de carvalho) aos 150 dias de envelhecimento

Para a amostra A4, após 150 dias de envelhecimento, o atributo mais preferido foi o sabor, com $52 \%$ dos provadores e notas entre 7,1 e 8 . Uma porcentagem de $48 \%$ dos provadores atribuiu notas entre 7,1 e 8 para a cor, seguida pelo aroma (44\%), com notas entre 8,1 e 9, e impressão global (40\%), com notas entre 8,1 e 9, conforme Figura 13. 


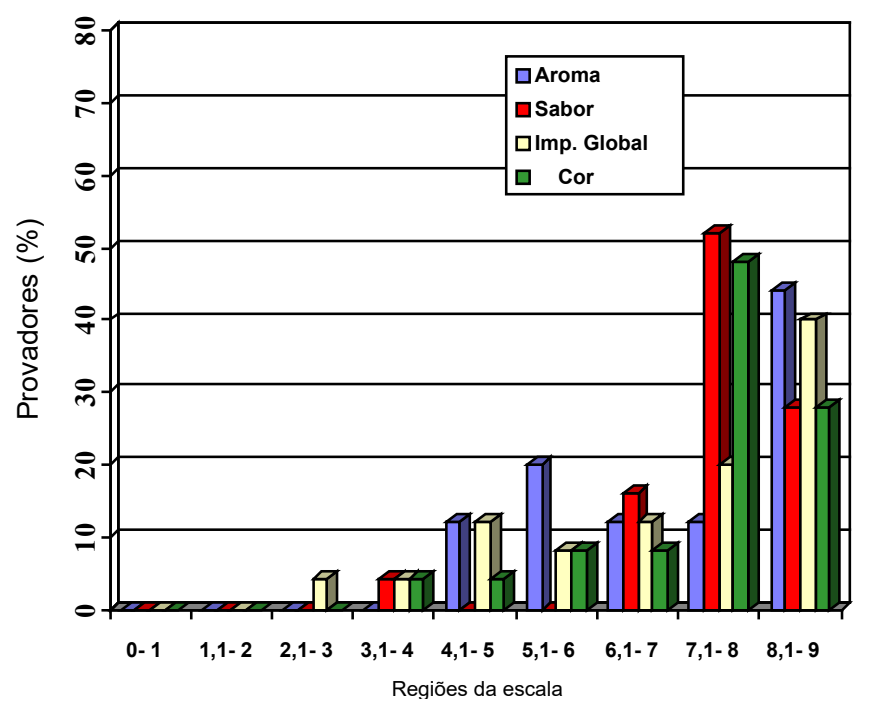

Figura 13 - Teste de aceitação da amostra A4 (armazenada em ancorote de carvalho irradiado) aos 150 dias de envelhecimento

No final do tempo de envelhecimento (180 dias), na amostra A2 foi analisada e constatada uma maior aceitação em relação ao aroma (76\%), com notas de 7,1 a 8, seguida por $60 \%$ de provadores que atribuíram notas de 8,1 a 9 para cor, $56 \%$ com notas entre 7,1 e 8 para impressão global, e $44 \%$ atribuíram notas de 7,1 a 8 para sabor, conforme observa-se na Figura 14.

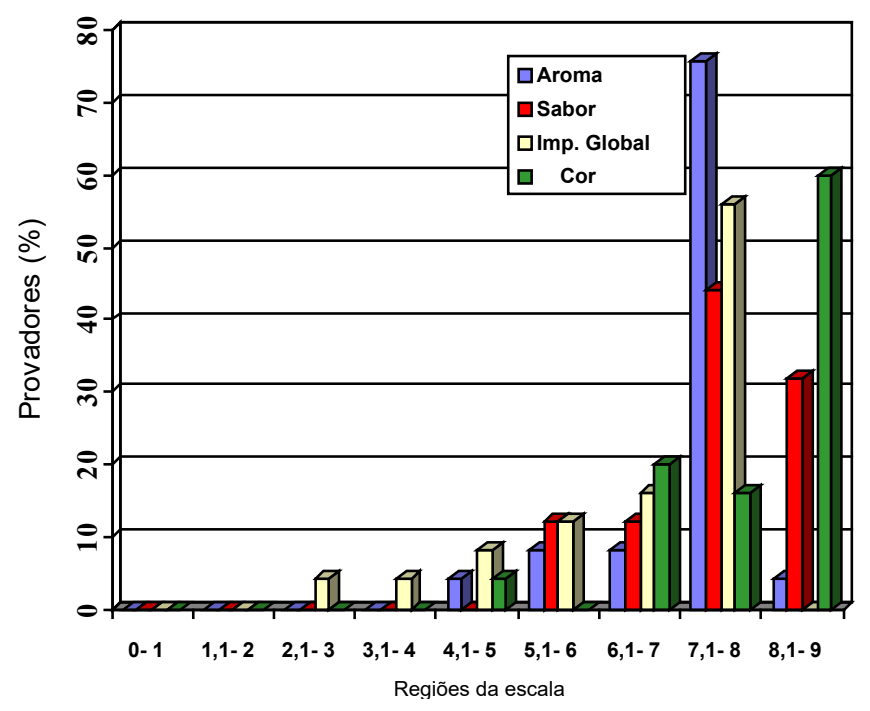

Figura 14 - Teste de aceitação da amostra A2 (armazenada em ancorote de carvalho) aos 180 dias de envelhecimento

Após 180 dias, a amostra A4, aguardente mantida em ancorote de carvalho irradiado, recebeu notas máximas ( 8,1 a 9) para cor por um número maior de provadores (76\%), seguida de $60 \%$ de preferência para aroma e 56\% para impressão global, com notas entre 7,1 e 8 . Finalmente, o atributo 
sabor obteve $48 \%$ da preferência com notas entre 7,1 e 8, valor este também superior ao encontrado nas demais amostras estudadas, como pode ser visto na Figura 15.

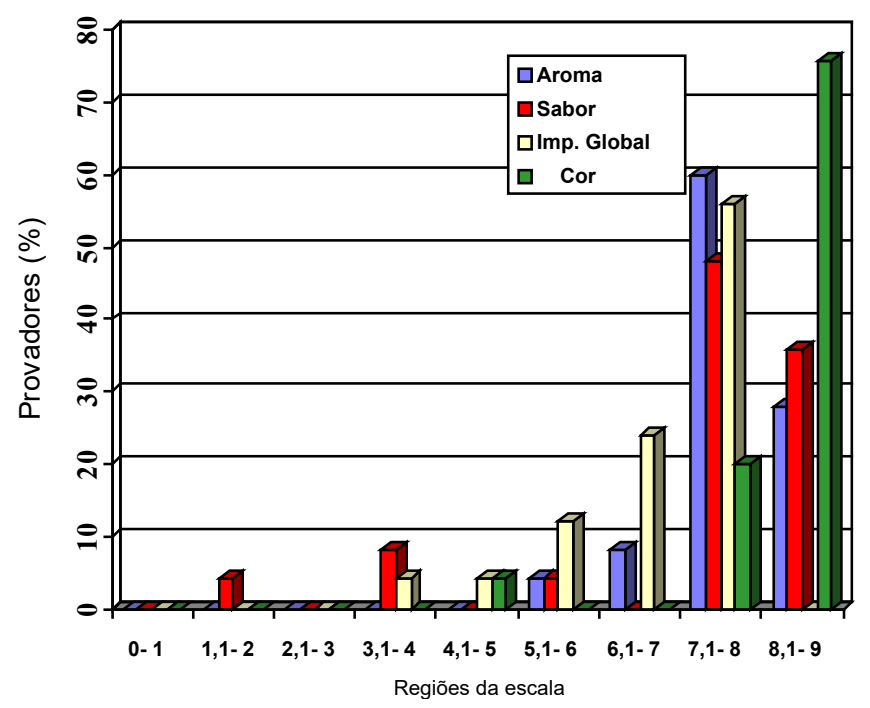

Figura 15 - Teste de aceitação da amostra A4 (armazenada em ancorote de carvalho irradiado) aos 180 dias de envelhecimento

Para a amostra A4 (armazenada em ancorote irradiado), observou-se maior aceitação, considerando os atributos aroma e cor que foram significativamente melhores no período de 30 a 180 dias em comparação com a amostra A2, submetida ao envelhecimento em ancorote isento de irradiação. Considerando-se a amostra A4, submetida a esse processo de envelhecimento rápido, observou-se o aperfeiçoamento do aroma o qual está associado à extração de substâncias presentes no ancorote de carvalho submetido à irradiação com raios gama que facilitam a extração do flavor, melhorando o aroma da bebida destilada. Esses resultados também podem ser comprovados conforme dados expressos na Tabela 4. 
Tabela 4 - Médias para comparação da aceitação das amostras em relação aos atributos aroma, sabor, impressão global e cor, em cada período avaliado $\|$

\begin{tabular}{|c|c|c|c|c|}
\hline \multicolumn{5}{|c|}{1 dia } \\
\hline Amostras & Aroma & Sabor & Impressão global & Cor \\
\hline 1 & $3,7966 a$ & $3,0897 \mathrm{a}$ & $3,4793 \mathrm{a}$ & $1,5690 \mathrm{~b}$ \\
\hline 2 & $4,1862 \mathrm{a}$ & $3,7069 \mathrm{a}$ & $4,0414 \mathrm{a}$ & $4,3759 \mathrm{a}$ \\
\hline 3 & $3,7926 \mathrm{a}$ & $3,2517 \mathrm{a}$ & $4,0448 \mathrm{a}$ & $1,3414 \mathrm{~b}$ \\
\hline 4 & $3,9793 \mathrm{a}$ & $3,8000 \mathrm{a}$ & $3,8448 \mathrm{a}$ & $4,8621 \mathrm{a}$ \\
\hline DMS & 0,8927 & 0,9198 & 0,9217 & 0,8320 \\
\hline \multicolumn{5}{|c|}{15 dias } \\
\hline Amostras & Aroma & Sabor & Impressão global & Cor \\
\hline 1 & $3,3280 \mathrm{~b}$ & $3,1320 \mathrm{~b}$ & $3,6600 \mathrm{a}$ & $1,5440 \mathrm{~b}$ \\
\hline 2 & $4,8600 \mathrm{a}$ & $4,4400 \mathrm{a}$ & $4,7240 \mathrm{a}$ & $6,3960 \mathrm{a}$ \\
\hline 3 & $3,5860 \mathrm{~b}$ & $3,2840 \mathrm{~b}$ & $3,9240 \mathrm{a}$ & $1,5040 \mathrm{a}$ \\
\hline 4 & $5,3720 \mathrm{a}$ & $4,3600 \mathrm{a}$ & $4,3240 \mathrm{a}$ & $6,4366 \mathrm{a}$ \\
\hline DMS & 1,1006 & 1,0644 & 1,3454 & 1,0843 \\
\hline \multicolumn{5}{|c|}{30 dias } \\
\hline Amostras & Aroma & Sabor & Impressão global & Cor \\
\hline 1 & $3,5640 \mathrm{c}$ & $3,0880 \mathrm{c}$ & $3,1880 \mathrm{c}$ & $1,3120 \mathrm{c}$ \\
\hline 2 & $5,1600 \mathrm{~b}$ & $4,3520 \mathrm{~b}$ & $4,7720 \mathrm{~b}$ & $5,2680 \mathrm{~b}$ \\
\hline 3 & $3,6400 \mathrm{c}$ & $3,0920 \mathrm{c}$ & $3,3480 \mathrm{c}$ & $1,2400 \mathrm{c}$ \\
\hline 4 & $6,4480 a$ & $5,4920 a$ & $5,9260 \mathrm{a}$ & $6,4680 \mathrm{a}$ \\
\hline DMS & 0,9201 & 0,9843 & 1,0377 & 1,1832 \\
\hline \multicolumn{5}{|c|}{60 dias } \\
\hline Amostras & Aroma & Sabor & Impressão global & Cor \\
\hline 1 & $3,3160 \mathrm{c}$ & $3,1200 \mathrm{~b}$ & $3,2440 \mathrm{~b}$ & $1,0960 \mathrm{~b}$ \\
\hline 2 & $5,5680 \mathrm{~b}$ & $4,6880 a$ & $4,8520 \mathrm{a}$ & $6,9920 \mathrm{a}$ \\
\hline 3 & $3,3520 \mathrm{c}$ & $3,2600 \mathrm{~b}$ & $3,4000 \mathrm{~b}$ & $1,1360 \mathrm{~b}$ \\
\hline 4 & $6,9160 a$ & $5,7360 \mathrm{a}$ & $5,9800 \mathrm{a}$ & $7,4320 a$ \\
\hline DMS & 1,2040 & 1,1245 & 1,1992 & 0,5798 \\
\hline \multicolumn{5}{|c|}{90 dias } \\
\hline Amostras & Aroma & Sabor & Impressão global & Cor \\
\hline 1 & $3,9160 \mathrm{c}$ & $3,8880 \mathrm{~b}$ & $3,9440 \mathrm{~b}$ & $1,0440 \mathrm{c}$ \\
\hline 2 & $6,0400 \mathrm{~b}$ & $5,9880 \mathrm{a}$ & $6,0880 \mathrm{a}$ & $6,5840 \mathrm{~b}$ \\
\hline 3 & $3,9720 \mathrm{c}$ & $3,7080 \mathrm{~b}$ & $4,3720 \mathrm{~b}$ & $1,1520 \mathrm{c}$ \\
\hline 4 & $7,3080 \mathrm{a}$ & $6,3760 a$ & $6,7440 \mathrm{a}$ & $7,8760 \mathrm{a}$ \\
\hline DMS & 1,2593 & 1,4682 & 1,2513 & 0,7566 \\
\hline \multicolumn{5}{|c|}{120 dias } \\
\hline Amostras & Aroma & Sabor & Impressão global & Cor \\
\hline 1 & $4,0920 \mathrm{c}$ & $3,6000 \mathrm{~b}$ & $3,3720 \mathrm{~b}$ & $1,0480 \mathrm{c}$ \\
\hline 2 & $5,4520 \mathrm{~b}$ & $6,4320 a$ & $5,9640 \mathrm{a}$ & $6,7800 \mathrm{~b}$ \\
\hline 3 & $3,8160 \mathrm{c}$ & $4,3960 \mathrm{~b}$ & $3,5520 \mathrm{~b}$ & $1,1200 \mathrm{c}$ \\
\hline 4 & $7,8360 \mathrm{a}$ & $6,1960 \mathrm{a}$ & $7,0640 \mathrm{a}$ & $7,5840 \mathrm{a}$ \\
\hline DMS & 1,1344 & 1,1342 & 1,3461 & 0,7673 \\
\hline \multicolumn{5}{|c|}{150 dias } \\
\hline Amostras & Aroma & Sabor & Impressão global & Cor \\
\hline 1 & $3,6520 \mathrm{~b}$ & $3,2400 \mathrm{~b}$ & $3,1720 \mathrm{~b}$ & $1,1680 \mathrm{c}$ \\
\hline 2 & $6,0920 \mathrm{a}$ & $7,0320 \mathrm{a}$ & $5,8040 \mathrm{~b}$ & $6,7800 \mathrm{~b}$ \\
\hline 3 & $3,2960 \mathrm{~b}$ & $3,3520 \mathrm{~b}$ & $3,3520 \mathrm{~b}$ & $1,2800 \mathrm{c}$ \\
\hline 4 & $7,1560 \mathrm{a}$ & $7,5560 \mathrm{a}$ & $6,9440 \mathrm{a}$ & $7,5840 \mathrm{a}$ \\
\hline \multirow[t]{2}{*}{ DMS } & 1,4019 & 1,0602 & 1,0618 & 0,7508 \\
\hline & & & & 180 dias \\
\hline Amostras & Aroma & Sabor & Impressão global & Cor \\
\hline 1 & $3,5640 \mathrm{~b}$ & $3,3920 \mathrm{c}$ & $3,3520 \mathrm{~b}$ & $1,0280 \mathrm{~b}$ \\
\hline 2 & $7,2720 \mathrm{a}$ & $7,5000 \mathrm{a}$ & $6,5800 \mathrm{a}$ & $7,8600 \mathrm{a}$ \\
\hline 3 & $3,0600 \mathrm{~b}$ & $3,0600 \mathrm{~b}$ & $3,6560 \mathrm{~b}$ & $1,1680 \mathrm{~b}$ \\
\hline 4 & $7,6640 \mathrm{a}$ & $7,2840 \mathrm{a}$ & $6,7800 a$ & $8,3000 \mathrm{a}$ \\
\hline DMS & 0,9709 & 0,9437 & 1,1754 & 0,5874 \\
\hline
\end{tabular}

" Médias com letras iguais na mesma coluna não diferem entre si significativamente; DMS= diferença mínima significativa. 


\section{CONCLUSÃO}

De acordo com os resultados obtidos nos testes sensoriais, pode-se afirmar que a irradiação do ancorote de carvalho favoreceu o desenvolvimento das qualidades sensoriais desejáveis da aguardente de cana durante o processo de envelhecimento em maior intensidade que o observado no ancorote não irradiado. Considerando que esse processo de envelhecimento em ancorote irradiado foi realizado em 180 dias, deve-se considerar a redução desse prazo comparado aos processos tradicionais, que levam 24 meses, e isso reflete em maior economia, menos utilização de barris de carvalho e maior rendimento, em decorrência da redução da evaporação do produto durante a estocagem.

A irradiação aplicada diretamente na amostra de aguardente não foi suficiente para causar alterações sensoriais perceptíveis nas condições experimentais avaliadas.

Assim, considera-se uma alternativa válida a possibilidade de utilização da radiação ionizante para acelerar o processo de envelhecimento de aguardente de cana-de-açúcar em curto prazo.

\section{REFERÊNCIAS}

1. Brasil. Decreto n. 2.314, de 04 setembro de 1997. Regulamenta a Lei n. 8.918, de 14 de julho de 1994, que dispõe sobre a padronização, a classificação, o registro, a inspeção, a produção e a fiscalização de bebidas. Diário Oficial [da] República Federativa do Brasil. Brasília, DF; 1997.

2. Bortoletto AM, Silvello GC, Alcarde AR. Chemical and microbiological quality of sugar cane juice influences the concentration of ethyl carbamate and volatile congeners in cachaça. Journal of The Institute of Brewing. 2015;121(2):251-6. doi: 10.1002/jib

3. Santiago WD, Cardoso MG, Santiago JL, Teixeira ML, Barbosa RB, Zacaroni LM, et al. Physicochemical profile and determination of volatile compounds in cachaça stored in new oak (Quercus sp.), amburana (Amburana cearensis), jatoba (Hymenaeae carbouril), balsam (Myroxylon peruiferum) and peroba (Paratecoma peroba) casks by SPME-GC-MS. Journal of Institute of Brewing. 2016;122(4):624-34. doi: 10.1002/jib.372

4. Bortoletto AM, Alcarde AR. Aging marker profile in cachaça is influenced by toasted oak chips. Journal Institute of Brewing. 2015;121(1):70-7. doi: 10.1002/jib.202

5. Conner JM, Piggott JR. Whiskies. In: Andrew GHL, Piggott JR, editors. Fermented Beverage Production. US: Springer; 2003. p. 239-62.

6. Lee KYM, Paterson A, Piggot JR, Richardson GD. Sensory discrimination of blended Scotch whiskies of different product categories. Food qual. pref. 2001;12(2):109-17.

7. Souza MDCA, Del Mastro NL. Irradiation of cane sugar spirit. Radiat. Phys. Chem. 2000;57(36):257-9.

8. Farkas JF, Farkas CM. Review: History and future of food irradiation. Trends food sci. Technol. 2012;22(2):121-6. doi: 10.1016/j.tifs.2010.04.002 
9. Internacional Consultative Group on Food Irradiaton. Facts about Food Irradiation. A series of Fact Sheets from the International Consultative Group on Food Irradiation (ICGFI). [Internet]. Viena, Áustria; 1999. [acesso em 2017 jun. 15]. Disponível em: https://www.nrc.gov/docs/ ML0611/ML061170095.pdf

10. Modanez L. Aceitação de alimentos irradiados: uma questão de educação [tese]. São Paulo: Universidade de São Paulo, Instituto de Pesquisas Energéticas e Nucleares; 2012.

11. SAS Institute. SAS User's Guide: statistics. Cary, NC: SAS Institute; 1993.

12. Moraes JS. Estudo comparativo das características químicas e sensoriais do rum e da cachaça [dissertação]. Araraquara: Universidade Estadual Paulista Julio de Mesquita Filho, Faculdade de Ciências Farmacêuticas; 2004.

13. Babior BM. Superoxide: a two-edged sword. Braz. J. Med. Biol. Res. 1997;30(2):141-55. doi: 10.1590/S0100-879X1997000200001

14. Dzhanpoladyan LM, Petrosyan TSL. Method for producing agent for acceleration of brandy maturation. USSR: Patent; 1971. 